\title{
QUEEN'S
UNIVERSITY
BELFAST
}

\section{Radiation-Pressure Acceleration of Ion Beams Driven by Circularly Polarized Laser Pulses}

Henig, A., Steinke, S., Schnürer, M., Sokollik, T., Hörlein, R., Kiefer, D., Jung, D., Schreiber, J., Hegelich, B. M., Yan, X. Q., Meyer-ter-Vehn, J., Tajima, T., Nickles, P. V., Sandner, W., \& Habs, D. (2009). Radiation-Pressure Acceleration of Ion Beams Driven by Circularly Polarized Laser Pulses. Physical Review Letters, 103(24), [245003]. https://doi.org/10.1103/PhysRevLett.103.245003

Published in:

Physical Review Letters

Document Version:

Publisher's PDF, also known as Version of record

Queen's University Belfast - Research Portal:

Link to publication record in Queen's University Belfast Research Portal

Publisher rights

Copyright 2009 The American Physical Society

\section{General rights}

Copyright for the publications made accessible via the Queen's University Belfast Research Portal is retained by the author(s) and / or other copyright owners and it is a condition of accessing these publications that users recognise and abide by the legal requirements associated with these rights.

Take down policy

The Research Portal is Queen's institutional repository that provides access to Queen's research output. Every effort has been made to ensure that content in the Research Portal does not infringe any person's rights, or applicable UK laws. If you discover content in the Research Portal that you believe breaches copyright or violates any law, please contact openaccess@qub.ac.uk. 


\title{
Radiation-Pressure Acceleration of Ion Beams Driven by Circularly Polarized Laser Pulses
}

\author{
A. Henig, ${ }^{1,2, *}$ S. Steinke, ${ }^{3}$ M. Schnürer, ${ }^{3}$ T. Sokollik ${ }^{3}$ R. Hörlein,,${ }^{1,2}$ D. Kiefer, ${ }^{1,2}$ D. Jung,,${ }^{1,2}$ J. Schreiber, ${ }^{1,2,4}$ \\ B. M. Hegelich, ${ }^{2,5}$ X. Q. Yan, ${ }^{1,6, \dagger}$ J. Meyer-ter-Vehn, ${ }^{1}$ T. Tajima, ${ }^{2,7}$ P. V. Nickles, ${ }^{3}$ W. Sandner, ${ }^{3}$ and D. Habs ${ }^{1,2}$ \\ ${ }^{1}$ Max-Planck-Institut für Quantenoptik, D-85748 Garching, Germany \\ ${ }^{2}$ Department für Physik, Ludwig-Maximilians-Universität München, D-85748 Garching, Germany \\ ${ }^{3}$ Max-Born-Institut, D-12489 Berlin, Germany \\ ${ }^{4}$ Plasma Physics Group, Blackett Laboratory, Imperial College London, SW7 2BZ, United Kingdom \\ ${ }^{5}$ Los Alamos National Laboratory, Los Alamos, New Mexico 87545, USA \\ ${ }^{6}$ State Key Lab of Nuclear Physics and Technology, Peking University, 100871, Beijing, China \\ ${ }^{7}$ Photomedical Research Center, JAEA, Kyoto, Japan \\ (Received 20 August 2009; published 9 December 2009)
}

\begin{abstract}
We present experimental studies on ion acceleration from ultrathin diamondlike carbon foils irradiated by ultrahigh contrast laser pulses of energy $0.7 \mathrm{~J}$ focused to peak intensities of $5 \times 10^{19} \mathrm{~W} / \mathrm{cm}^{2}$. A reduction in electron heating is observed when the laser polarization is changed from linear to circular, leading to a pronounced peak in the fully ionized carbon spectrum at the optimum foil thickness of $5.3 \mathrm{~nm}$. Two-dimensional particle-in-cell simulations reveal that those $\mathrm{C}^{6+}$ ions are for the first time dominantly accelerated in a phase-stable way by the laser radiation pressure.
\end{abstract}

DOI: 10.1103/PhysRevLett.103.245003

PACS numbers: 52.38.Kd, 41.75.Jv, 52.50.Jm, 52.65.Rr

The generation of highly energetic ion beams from laserplasma interactions has attracted great interest since the pioneering work that was carried out $10-15$ years ago [15]. In the vast majority of previous studies, foil targets ranging in thickness from a few to several tens of microns have been irradiated by linearly polarized, intense $\left(10^{18}-10^{21} \mathrm{~W} / \mathrm{cm}^{2}\right)$ laser pulses. Here, target normal sheath acceleration (TNSA) was found to be the predominant mechanism leading to the emission of multi-MeV, high-quality ion beams. In TNSA, protons are preferentially accelerated from the nonirradiated surface of the opaque foil, resulting in a continuous, exponential spectrum with cutoff energy up to $60 \mathrm{MeV}$ [6]. By decreasing the foil thickness to values only slightly above the decay length of the evanescent field, an enhancement in the acceleration of heavier ions was obtained recently [7]. The possibility to accelerate quasimonoenergetic ion bunches has already been demonstrated within the TNSA regime by restricting the ion source to a small volume where the sheath field is homogenous [8-10]. However, this method suffers from a very low conversion efficiency.

Recently, a new mechanism for laser-driven ion acceleration was proposed, where particles gain energy directly from the radiation pressure (RP) exerted onto the target by the laser beam [11-18], an idea that goes back to [19]. For RPA to become dominant, a thin foil is irradiated by a circularly polarized laser pulse at normal incidence. Owing to the absence of an oscillating component in the $\vec{v} \times \vec{B}$ force, electron heating is strongly suppressed. Instead, electrons are compressed to a highly dense electron layer piling up in front of the laser pulse which in turn accelerates ions. By choosing the laser intensity, target thickness, and density such that the radiation pressure equals the restoring force given by the charge separation field, the whole focal volume eventually propagates ballistically as a quasineutral plasma bunch, continuously gaining energy from the laser field. In this scenario, all particle species are accelerated to the same velocity, which intrinsically results in a monochromatic spectrum. As long as the electron temperature is kept low, a phase-stable acceleration can be maintained, and the process is expected to lead to very high conversion efficiencies and ion energies scaling linearly with laser intensity under optimum conditions.

Despite the anticipated highly promising characteristics of RPA, according to our knowledge experimental investigation of ion acceleration using circular polarization has only been carried out at intensities smaller than $4 \times$ $10^{18} \mathrm{~W} / \mathrm{cm}^{2}$ and targets of several micron thickness irradiated at oblique incidence [20,21], thus being far off the parameters necessary for radiation pressure to become the dominant acceleration mechanism.

In this Letter, we present for the first time experimental studies of ion acceleration driven by circularly polarized laser pulses at significantly increased intensities of $5 \times$ $10^{19} \mathrm{~W} / \mathrm{cm}^{2}$, corresponding to a radiation pressure of 30 Gbar. Diamondlike carbon (DLC) foils of thickness 2.9-40 $\mathrm{nm}$ have been irradiated at normal incidence and ultrahigh laser pulse contrast. When compared to the case of linear polarization, we observe a pronounced decrease in the number of hot electrons generated. While for linear polarization the spectra of all ion species decay monotonically up to a certain cutoff value, a distinct peak of energy $30 \mathrm{MeV}$ emerges in the spectrum of fully ionized carbon ions at the optimum target thickness of $(5.3 \pm 1.3) \mathrm{nm}$ and circular polarization. Two-dimensional particle-in-cell (PIC) simulations confirm that under these conditions 
$\mathrm{C}^{6+}$ ions are dominantly accelerated by the laser radiation pressure at values up to $\sim 10^{21} \mathrm{~m} / \mathrm{s}^{2}$.

The described experiments have been carried out at the 30 TW Ti:sapphire laser system located at Max Born Institute, delivering $1.2 \mathrm{~J}$ of energy stored in pulses of $\tau=$ $45 \mathrm{fs}$ FWHM duration at a central wavelength of $\lambda=$ $810 \mathrm{~nm}$. The relative intensity of prepulses and of the amplified spontaneous emission (ASE) pedestal was characterized by means of a 3rd order autocorrelator to be smaller than $10^{-7}$ at times earlier than -10 ps prior to the arrival of the main peak. In order to further enhance that value, a recollimating double plasma mirror [22,23] was introduced into the laser beam path, resulting in an estimated contrast of $\sim 10^{-11}$. Taking into account the measured $60 \%$ energy throughput of this setup, $\sim 0.7 \mathrm{~J}$ were focused by an $f / 2.5$ off axis parabolic mirror to a FWHM diameter focal spot size of $2 w_{0}=3.6 \mu \mathrm{m}$. A peak intensity of $I_{0}=5 \times 10^{19} \mathrm{~W} / \mathrm{cm}^{2}$ was achieved, corresponding to a normalized laser vector potential maximum of $a_{0}=5$ for linear and $a_{0}=5 / \sqrt{2}=3.5$ for circular polarization. To vary the laser polarization to circular, a mica crystal operating as $\lambda / 4$ wave plate was introduced into the beam path behind the plasma mirror setup.

DLC foils of thickness $d=2.9-40 \mathrm{~nm}$ and density $\rho=$ $2.7 \mathrm{~g} / \mathrm{cm}^{3}$ were placed in the focal plane at normal incidence. Compared to other material available, DLC offers unique properties for mechanically stable, ultrathin, freestanding targets, such as exceptionally high tensile strength, hardness, and heat resistance, owing to the high fraction of $s p^{3}$, i.e., diamondlike bonds of $\sim 75 \%$. The thickness of the DLC foils was characterized by means of an atomic force microscope (AFM), including the hydrocarbon contamination layer on the target surface which was present during the experiments. In addition, the depthdependent composition of the target was measured via elastic recoil detection analysis (ERDA). From these measurements we obtain a thickness of $\sim 1 \mathrm{~nm}$ for the hydrocarbon contamination layer. Throughout this Letter we are referring to the combined thickness of bulk and surface layer as it appears in the actual ion acceleration experiment presented.

To characterize the accelerated ions, a Thomson parabola spectrometer was placed at a distance of $\sim 0.5 \mathrm{~m}$ (solid angle $\sim 1.14 \times 10^{-7} \mathrm{sr}$ ) along the laser propagation, i.e., target normal direction. Ion traces were detected at the back of the spectrometer by a CCD camera coupled to a microchannel plate (MCP) with phosphor screen [24]. In addition, a magnetic electron spectrometer (solid angle $\sim 2 \times 10^{-4}$ sr) equipped with Fujifilm BAS-TR image plates was positioned behind the target at an angle of $22.5^{\circ}$ with respect to the laser axis.

The obtained maximum ion energies per atomic mass unit plotted over target thickness $d$ are shown in Fig. 1(a) for linear and circular polarization. While linearly polarized irradiation yields higher proton and carbon energies, a strong dependence on initial foil thickness is visible in both
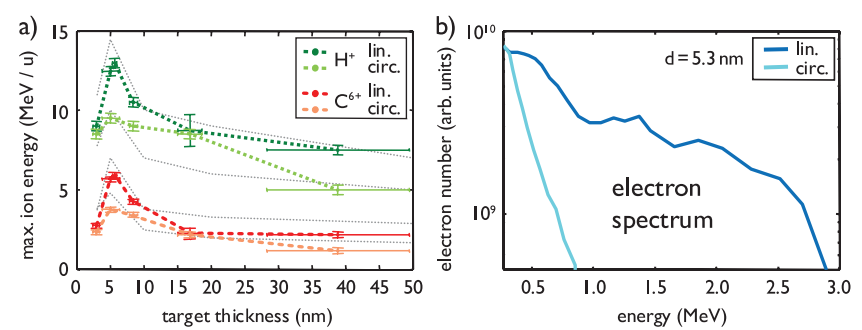

FIG. 1 (color). (a) Experimentally observed maximum proton (green dotted line, light green dotted line) and carbon $\mathrm{C}^{6+}$ (red dotted line, orange dotted line) energies per atomic mass unit over target thickness for linearly and circularly polarized irradiation. Gray dotted lines represent the respective values obtained from 2D PIC simulations. Corresponding electron spectra measured at the optimum target thickness $d=5.3 \mathrm{~nm}$ are given in (b), showing a strong reduction in electron heating for circularly polarized irradiation.

cases, with a distinct optimum at $d=5.3 \mathrm{~nm}$. Using circular polarization, the value of the optimum foil thickness is theoretically expected to be given by the condition $a_{0} \simeq$ $\sigma$ [16]; i.e., the dimensionless laser vector potential $a_{0}$ approximately equals the normalized areal density $\sigma=$ $\left(n_{e} / n_{\mathrm{cr}}\right)(d / \lambda)$ of the target. Here, $n_{e}$ stands for the electron density, whereas $n_{\mathrm{cr}}=\epsilon_{0} m_{e} \omega_{L}^{2} / e^{2}$ denotes the critical density of the plasma with electron mass $m_{e}$ and laser carrier frequency $\omega_{L}$. This prevision is in excellent agreement with our experimental result of $3.5=a_{0} \simeq \sigma=3.3$. At the optimum target thickness, maximum energies for protons and carbon ions of 10 and $45 \mathrm{MeV}$ are generated for circular polarization, while linear polarization gives 13 and $71 \mathrm{MeV}$, respectively. The corresponding electron spectra for $d=5.3 \mathrm{~nm}$ are shown in Fig. 1(b). It can be clearly seen that circularly polarized irradiation results in a pronounced reduction in the number of highly energetic electrons as expected. To illustrate the consequent impact on the acceleration of ions, experimentally observed proton and carbon spectra are plotted in Figs. 2(a) and 2(b) for linear and circular polarization at the optimum foil thickness. A monotonically decaying spectrum is obtained for both protons and carbon ions in the case of linear polarization. In contrast, when the laser polarization is changed to circular the spectrum of fully ionized carbon $\mathrm{C}^{6+}$ atoms [Fig. 2(b)] reveals two components. In addition to the continuously decreasing low energetic ion population reaching up to $\sim 20 \mathrm{MeV}$, a distinct peak is seen at higher energies, centered around $30 \mathrm{MeV}$. We deduce a conversion efficiency of $\sim 2.5 \%$ to $\mathrm{C}^{6+}$ ions within the peak energy interval $20-40 \mathrm{MeV}$ and a beam of half angle $\sim 9^{\circ}$, in good agreement with the corresponding value predicted by $\eta=2 I_{0} \tau /\left(c^{2} \rho d\right) \simeq 3.7 \%$ [14]. The spectral spike of $\mathrm{C}^{6+}$ ions was repeatedly observed in consecutive shots at the optimum foil thickness of $5.3 \mathrm{~nm}$ and circular polarization, whereas the shape of the proton spectrum was not affected when varying the polarization. 

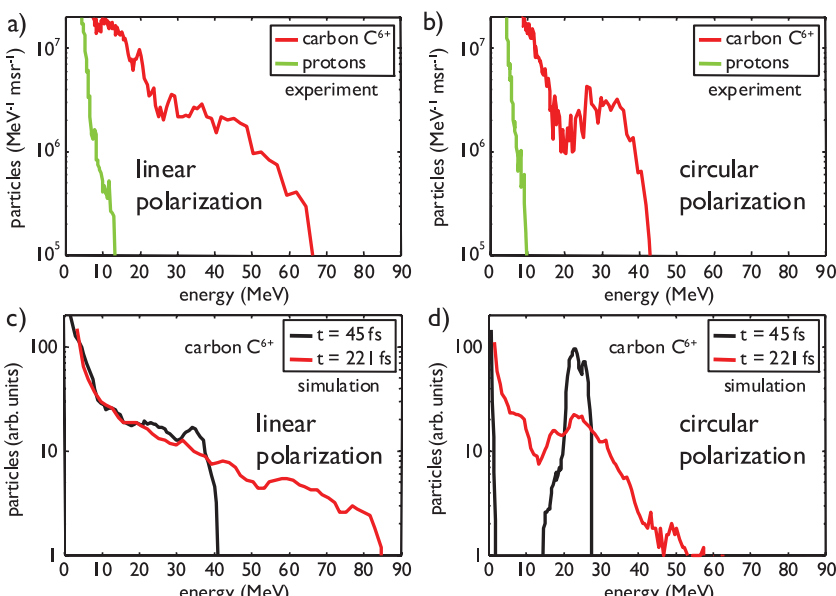

FIG. 2 (color). Experimentally observed proton (green curves) and carbon $\mathrm{C}^{6+}$ (red curves) spectra in the case of linear (a) and circular (b) polarized irradiation of a $5.3 \mathrm{~nm}$ thickness DLC foil. The corresponding curves as obtained from 2D PIC simulations (c),(d) show excellent agreement with the measured distributions at late times (red curves, $t=221 \mathrm{fs}$ after the arrival of the laser pulse maximum at the target). A quasimonoenergetic peak generated by radiation-pressure acceleration is revealed for circular polarization, being still isolated at the end of the lasertarget interaction (black curve, $t=45 \mathrm{fs}$ ).

In order to support our experimental findings, twodimensional PIC simulations were carried out. The DLC foil targets were modeled by a solid density $\left(n_{e} / n_{\mathrm{cr}}=\right.$ 500 ), rectangularly shaped plasma slab of zero initial electron temperature, composed of $90 \% \mathrm{C}^{6+}$ ions and $10 \%$ protons in number density. The laser pulse is of Gaussian shape in both the spatial distribution in the focal plane as well as in time, with a FWHM diameter of $4 \mu \mathrm{m}$ and a FWHM duration of $45 \mathrm{fs}$, resulting in a peak intensity of $I_{0}=5 \times 10^{19} \mathrm{~W} / \mathrm{cm}^{2}$. A simulation domain of size $10 \mu \mathrm{m}$ in transverse $(y)$ and $20 \mu \mathrm{m}$ in longitudinal $(x)$ dimension was used, subdivided into a grid of $1200 \times$ 10000 cells each occupied by 2000 particles. In the following, PIC simulation times are given relative to $t=0$, when the peak of the laser pulse reaches the initial position of the target $(x=3 \lambda)$.

The calculated carbon spectra are presented in Figs. 2(c) and 2(d) for linearly and circularly polarized irradiation. To account for the small solid angle of observation of the Thomson parabola spectrometer, only particles propagating in forward direction within a cone of half angle $0.01 \mathrm{rad}$ were considered for the simulated graphs. A continuously decaying spectrum is generated in the case of linear polarization, which agrees well with the experimental result [Figs. 2(a) and 2(c)]. This scenario changes drastically when circular polarization is used. As shown in Fig. 2(d), an isolated, quasimonoenergetic peak emerges in this configuration at the end of the laser-target interaction (black line, $t=45 \mathrm{fs}$ ). In the carbon ion phase space [Fig. 3(b)], a significant amount of particles is located in a discrete area, constituting a rotating structure. The series of loops origi-
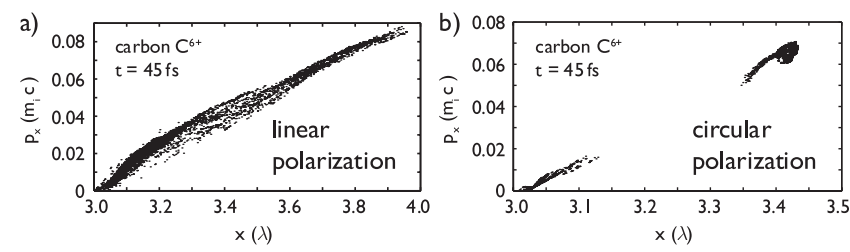

FIG. 3. Carbon ion phase space at the end of the laser-target interaction ( $t=45 \mathrm{fs}$ ). A significant amount of particles form a distinct loop in the case of circular polarization (b), giving evidence of a phase-stable acceleration driven by the laser radiation pressure.

nates from the continuing front side acceleration and the ballistic evolution of the target, thus giving clear evidence of radiation pressure to be the dominant acceleration force [11-18]. This is in strong contrast to the use of linear polarization, where at that moment the carbon ion phase space already forms a continuous, straight line [Fig. 3(a)]. The striking difference in the acceleration dynamics can also be directly inferred when examining the electron and ion density distributions as observed in our simulations (Fig. 4). For use of circular polarization, the electron population maintains its structure as a thin layer of high density being pushed by the laser in forward direction. As a consequence, carbon ions copropagate with the compressed electron cloud and the whole focal volume is accelerated as a quasineutral dense plasma bunch by the laser radiation pressure. Since all ion species move at equal velocity, RPA-driven protons attain only $30 / 12=2.5 \mathrm{MeV}$ energy, thus not being visible in the continuous experimental spectrum reaching up to $\sim 10 \mathrm{MeV}$ [Fig. 2(b)]. This scenario is contrary to the case of linear polarization [Figs. 4(a) and 4(c)], where the foil electrons are heavily heated by the laser. Accordingly, electrons have already
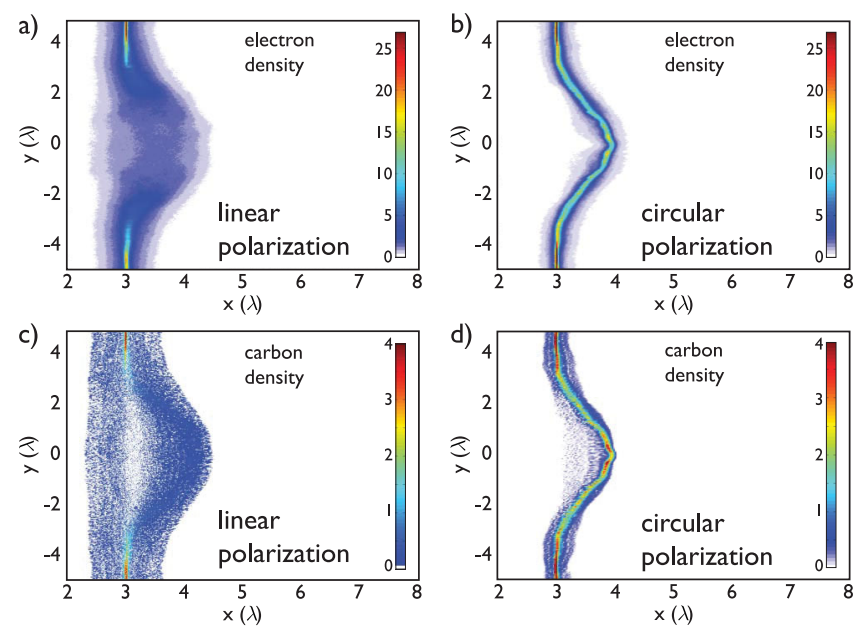

FIG. 4 (color). Cycle-averaged electron (a),(b) and carbon ion (c),(d) density at $t=61 \mathrm{fs}$ after the peak of the laser pulse reached the $5.3 \mathrm{~nm}$ target initially located at $x=3 \lambda$. While linear polarization results in strong expansion of the target caused by hot electrons, for circularly polarized irradiation the foil is accelerated as a dense, quasineutral plasma bunch. 
spread significantly and the electron density in the focal spot center, where electrons gain their highest energies, is considerably reduced. Although the acceleration of carbon ions is still asymmetric, favoring the laser forward direction (see also [7]), it is dominated by the sheath field of the expanding electrons.

However, given the laser parameters used in the presented experiment, the narrow isolated quasimonoenergetic peak in the carbon spectrum as it is present right after the end of laser-target interaction does not preserve its shape upon further propagation of the ion beam [Figs. 2(b) and 2(d)]. Even though the apex energy stays constant, the spectral distribution broadens and partially merges with the low energetic ion population which at this point still gains energy, resulting in the carbon ion spectrum as observed experimentally. This behavior can be attributed to the considerable deformation of the foil plasma by the tightly focused Gaussian laser spot [Figs. 4(b) and 4(d)]. Owing to the thus no longer normal but rather oblique incidence on the bent plasma surface, particularly at the end of the lasertarget interaction, perpendicular electric field components are present. Those efficiently heat electrons located in the warped spatial regions, which then quickly spread around the target, causing the monoenergetic peak in the carbon spectrum to broaden as well as ions in the low energetic part of the distribution to gain further energy and close the gap to the RPA-generated population. We note that this temporal evolution of the foil plasma shape was already discussed theoretically in [14], where in order to prevent the distortion of the ion spectrum an upper limit for the laser pulse duration is given by $t_{\max } \simeq \sqrt{2 w_{0} c \rho d / I_{0}}=$ $176 \mathrm{fs}$. Even though the laser pulse duration used in the presented experimental study is significantly shorter than $t_{\max }$, a spectral deformation is still observed, taking place after the end of the laser-target interaction. In the near future, this might be compensated for by properly shaping the laser focal spot distribution [25] and/or the nanometerthin foil target [26]. Additionally, increasing the intensity to $7 \times 10^{21} \mathrm{~W} / \mathrm{cm}^{2}$ is expected to result in a stabilization of the monoenergetic feature due to plasma instabilities [27], while beyond $10^{23} \mathrm{~W} / \mathrm{cm}^{2} \mathrm{RPA}$ is predicted to become dominant even for linear polarization with a laser-toion conversion efficiency approaching unity in the highly relativistic limit [28].

In summary, we have presented experimental investigations on ion acceleration from nanometer-thin DLC foil targets irradiated by linearly and circularly polarized, highly intense laser pulses. A strong decrease in the number of hot electrons is observed for use of circular polarization, resulting in a pronounced peak centered at $30 \mathrm{MeV}$ in the carbon $\mathrm{C}^{6+}$ ion spectrum at the optimum foil thickness of $5.3 \mathrm{~nm}$, which is in excellent agreement with the condition $a_{0} \simeq \sigma$ [16]. Compared to [8], we demonstrate a more than 40 times increase in conversion efficiency when considering an identical energy spread around the apex. Two-dimensional PIC simulations have been carried out, giving clear evidence that those ions are for the first time dominantly accelerated in a phase-stable way by the laser radiation pressure. Being recently widely studied in theory, our comparative measurements provide the first experimental proof of the feasibility of radiation-pressure acceleration to become the dominant mechanism for ion acceleration when circular polarization is used. These results are a major step towards highly energetic, monochromatic ion beams generated at high conversion efficiencies as demanded by many potential applications. Those include fast ignition inertial confinement fusion (ICF) as well as oncology and radiation therapy.

This work was supported by DFG through Transregio SFB TR18 and the DFG Cluster of Excellence MunichCentre for Advanced Photonics (MAP). A. H., D. K., and D. J. acknowledge financial support from IMPRS-APS, J. S. from DAAD, X. Q. Y. from the Humboldt foundation and NSFC(10855001).

*andreas.henig@mpq.mpg.de

†xyan@mpq.mpg.de

[1] A. P. Fews et al., Phys. Rev. Lett. 73, 1801 (1994).

[2] E. L. Clark et al., Phys. Rev. Lett. 85, 1654 (2000).

[3] A. Maksimchuk et al., Phys. Rev. Lett. 84, 4108 (2000).

[4] R. A. Snavely et al., Phys. Rev. Lett. 85, 2945 (2000).

[5] S. P. Hatchett et al., Phys. Plasmas 7, 2076 (2000).

[6] L. Robson et al., Nature Phys. 3, 58 (2007).

[7] A. Henig et al., Phys. Rev. Lett. 103, 045002 (2009).

[8] B. M. Hegelich et al., Nature (London) 439, 441 (2006).

[9] H. Schwoerer et al., Nature (London) 439, 445 (2006).

[10] S. Ter-Avetisyan et al., Phys. Rev. Lett. 96, 145006 (2006).

[11] A. Macchi et al., Phys. Rev. Lett. 94, 165003 (2005).

[12] X. Zhang et al., Phys. Plasmas 14, 073101 (2007).

[13] T. V. Liseikina et al., Appl. Phys. Lett. 91, 171502 (2007).

[14] O. Klimo et al., Phys. Rev. ST Accel. Beams 11, 031301 (2008).

[15] A. P. L. Robinson et al., New J. Phys. 10, 013021 (2008).

[16] X. Q. Yan et al., Phys. Rev. Lett. 100, 135003 (2008).

[17] S. G. Rykovanov et al., New J. Phys. 10, 113005 (2008).

[18] B. Qiao et al., Phys. Rev. Lett. 102, 145002 (2009).

[19] G. Marx, Nature (London) 211, 22 (1966).

[20] A. Fukumi et al., Phys. Plasmas 12, 100701 (2005).

[21] M. Kado et al., Laser Part. Beams 24, 117 (2006).

[22] G. Doumy et al., Phys. Rev. E 69, 026402 (2004).

[23] A. A. Andreev et al., Phys. Plasmas 16, 013103 (2009).

[24] S. Ter-Avetisyan et al., J. Phys. D 38, 863 (2005).

[25] M. Chen et al., Phys. Plasmas 15, 113103 (2008).

[26] M. Chen et al., Phys. Rev. Lett. 103, 024801 (2009).

[27] X. Q. Yan et al., Phys. Rev. Lett. 103, 135001 (2009).

[28] T. Esirkepov et al., Phys. Rev. Lett. 92, 175003 (2004). 